\title{
STRUCTURAL STUDIES OF CAPSULAR POLYSACCHARIDE OF TYPE XII Diplococcus pneumoniae*
}

Jodie L. DUKe, IRWIN J. GoldSTEIN,

Department of Biological Chemistry, The University of Michigan Medical School, Ann Arbor, Michigan 48104 (U.S. A.)

AND Joseph A. Crfoneli

La Rabida Institute, University of Chicago, and Department of Biochemistry, The University of Chicago, Chicago, Illinois 60637 (U. S. A.)

(Received February 8th, 1974; accepted April 29th, 1974)

\section{ABSTRACT}

The capsular polysaccharide of Diplococcus pneumoniae Type XII contains residues of $D$-glucose and $D$-galactose in a molar ratio of $2: 1$. The methylated polysaccharide yielded upon hydrolysis 2,3,4,6-tetra- and 3,4,6-tri- $O$-methyl-D-glucose and 2,3,4,6-tetra- $O$-methyl-D-galactose as the only neutral methyl sugars. Periodate oxidation of the polysaccharide resulted in destruction of all neutral sugars and immunochemical activity against rabbit antisera. Periodate oxidation of the methyl $O$-methylglycosides obtained after hydrolysis of the methylated polysaccharide indicated that at least $30 \%$ of the $L$-fucosamine residues are substituted at $C-4$ in the polysaccharide. It is concluded that the polysaccharide consists of a hexosamine backbone that is substituted by $D$-galactosyl and kojibiosyl side-chains. The proposed terminal D-galactosyl residues apparently are sterically hindered from interacting with several D-galactose-binding proteins.

\section{INTRODUCTION}

The capsular polysaccharide of Type XII Diplococcus pnetrmoniae (S XII) has been purified and shown to contain D-glucose, D-galactose, $N$-acetyl-L-fucosamine, and $N$-acetyl-D-galactosamine ${ }^{1}$. Smith degradation of the polysaccharide resulted in exclusive destruction of the neutral sugars and formation of glycerol. The results of the Smith degradation suggested a backbone of hexosamine residues with the ncutral sugars occurring in side-chains ${ }^{1}$. Recently, kojibiose (2-O- $\alpha$-D-glucopyranosylD-glucose) was isolated from partial acid hydrolysis of S XII and crystalline kojibiose octaacetate was obtained by acetolysis of the polysaccharide ${ }^{2}$. Immunochemical evidence had suggested ${ }^{3,4}$ that kojibiose is an important structural feature of $\mathrm{S}$ XII. This report presents further structural studies on S XII based on methylation and quantitative periodate oxidation. The results ascertain that $\mathrm{S}$ XII contains a

\footnotetext{
*Dedicated to the memory of Professor W. Z. Hassid.
} 
hexosamine backbone and contribute new information with regards to substitution by nonreducing terminal $\mathbf{D}$-galactose and kojibiose residues.

\section{EXPERIMENTAL}

Materials and methods. - The following substances were generous gifts: $\alpha$-galactosidases from Phasealus vulgaris and Aspergillus niger, Dr. $\mathrm{O}$. P. Bahl (State University, New York at Buffalo); $\beta$-galactosidase from bovine testes, Drs. G. Jourdian and J. Distler (The University of Michigan); $\alpha$-D-galactosyl-binding lectin from Bandeiraea simplicifolia, Dr. C. E. Hayes (The University of Michigan); 2-O- $\alpha$-D-galactopyranosyl-D-glucose, Dr. B. Helferich (Bonn, Germany); $N$-acetylD-fucosamine and $N$-acetyl-L-fucosamine, Dr. M. B. Perry (Ottawa, Ontario, Canada); 2,3-di- $O$-methyl-D-glucose and 3,4-di- $O$-methyl-D-glucose, Dr. B. Lewis (Cornell University, Ithaca, New York); and authentic standards of several methylated alditol acetates, Dr. A. Misaki (Osaka University, Osaka, Japan).

Neutral sugars were estimated by the phenol-sulfuric acid procedure ${ }^{5}$ and hexosamines were determined by a modification of the Elson-Morgan procedure ${ }^{6}$. Quantitative determinations of D-glucose and D-galactose were also performed with the Technicon carbohydrate autoanalyzer ${ }^{7}$.

Rabbit antisera to type XII Diplococcus pneumoniae were prepared with a lyophilized culture (American Type Culture Collection, Rockville, Maryland) essentially as described by Campbell et $a l^{8}$.

Methylation of S XII. - Type XII polysaccharide was purified by ion-exchange chromatography and ammonium sulfate precipitation according to Cifonelli et al. ${ }^{1}$. The preparation was dialyzed exhaustively against distilled water, lyophilized, and dried in vacuo for $4 \mathrm{~h}$ at $60^{\circ}$. Methylation studies were conducted by a modification of the Hakomori methylation procedure by use of methylsulfinyl carbanion and methyl iodide in dimethyl sulfoxide? ${ }^{9}$. The polysaccharide $(10 \mathrm{mg})$ was methylated 2 times, dialyzed overnight against tap water, and lyophilized. Acetolysis and acid hydrolysis of methylated S XII were performed under the conditions used by Stellner et al. ${ }^{10}$. The alditol acetate derivatives of the $O$-methylglycitols were separated by g.l.c. with a column packed with $3 \%$ ECNSS-M on Gas Chrom Q (Applied Science Labs, Inc., State College, Pa. 16801). Gas-liquid chromatography was performed on a combination g.l.c.-m.s. 30 double-beam mass spectrophotometer (Associated Electrical Industries, Barton Dock Road, Urmston, Manchester, England) equipped with a Pye series 104 chromatograph oven and temperature programmer. A resolution of 1,000 and an electron energy of $70 \mathrm{eV}$ were used with an interface temperature of 200-225 and a column-oven-temperature of $190^{\circ}$.

Methyl glycosides were prepared from methylated S XII by heating it in 2M methanolic hydrogen chloride ${ }^{11}$. The methyl glycosides were separated by a $F$ and $M$ Scientific 402 high-efficiency gas chromatograph (Hewlett-Packard, Avondale, Pa. 19311) on a column of $15 \%$ poly (1,4-butanediol succinate) on Neosorb N.C. (Applied Science Labs, Inc.) at $175^{\circ}$, according to the procedure of Aspinall ${ }^{12}$. 
Periodate oxidation. - Periodate consumption was determined by the iodimetric titration method of $\mathrm{Kabat}^{13}$. The polysaccharide $(8.9 \mathrm{mg}$, containing $18.5 \mu$ moles of neutral sugar) was dissolved in $24 \mathrm{~mm}$ sodium metaperiodate ( $25 \mathrm{ml}$ ). The solution and a control lacking S XII were incubated in the dark at $27^{\circ}$ until analysis indicated that the consumption of periodate had ceased $(100 \mathrm{~h})$. The reaction was terminated by addition of sodium borohydride $(100 \mathrm{mg})$ to destroy the excess of periodate and to reduce the oxidized polysaccharide. After incubation for $12 \mathrm{~h}$ at $25^{\circ}$, the degraded polysaccharide was dialyzed at $4^{\circ}$ against 100 volumes of distilled water. Lyophilization of the degraded polysaccharide gave $6 \mathrm{mg}$ of white material.

Preparation and oxidation of O-methylglycitols of methylated $S X I I$. - The polysaccharide (10 mg, containing $22 \mu$ moles of neutral sugar), methylated as just described, was incubated for $3 \mathrm{~h}$ at $4^{\circ}$ with $75 \%(\mathrm{w} / \mathrm{v})$ sulfuric acid $(0.2 \mathrm{ml})$ in order to solubilize it. After water $(1.4 \mathrm{ml})$ was added, the solution was heated for $6 . \mathrm{h}$ at $100^{\circ}$ and neutralized with barium carbonate. The precipitate was removed and sodium borohydride $(\sim 20 \mathrm{mg})$ was added to the filtrate. The reaction was allowed to proceed for $2 \mathrm{~h}$, the $\mathrm{pH}$ adjusted to 7.0 with $\mathrm{m}$ hydrochloric acid, and the solvent evaporated on a rotary evaporator. Water $(2.5 \mathrm{ml})$ was added to the flask and an aliquot analyzed for L-fucosamine content by the periodate oxidation method of Bhattacharyya and Aminoff ${ }^{14}$.

Quantitative hapten-inhibition analysis. - For quantitative hapten-inhibition analyses, such dilutions of whole sera were used that the antigen, in the absence of hapten, precipitated approximately $10 \mu \mathrm{g}$ of nitrogen; a total volume of $0.4 \mathrm{ml}$ was used for each tube. Hapten and antiserum were incubated for $1 \mathrm{~h}$ at $37^{\circ}$. After addition of S XII, the tubes were incubated for $1 \mathrm{~h}$ at $37^{\circ}$, and then for $7-10$ days at $4^{\circ}$. Precipitates were washed with ice-cold $0.01 \mathrm{M}$ phosphate buffer $(0.2 \mathrm{ml}, \mathrm{pH} 7.5)$. Total nitrogen was determined by ninhydrin analysis ${ }^{15}$.

RESULTS

Acid hydrolysis of $S X I I$. - The neutral sugar components of S XII were identified as D-glucose and D-galactose by their relative retention times on the Technicon carbohydrate autoanalyzer. The retention times, relative to D-mannose as the internal standard, were 1.48 and 1.26 for D-glucose and D-galactose, respectively. The molar ratio of $\mathrm{D}$-glucose to $\mathrm{D}$-galactose was 2.04:1.00. The release of hexosamine from S XII (30 $\mu \mathrm{g}$ containing 62 nmoles of neutral sugar) during hydrolysis with $2 \mathrm{M}$ hydrochloric acid at $100^{\circ}$ is shown in Table I. Hexosamine release attained a maximum value within $3 \mathrm{~h}$. The ratio of neutral sugar to hexosamine was 1.51:1.00, which is in agreement with an earlier report ${ }^{1}$. These data suggest that the molar ratio of $\mathrm{D}-$ glucose to D-galactose to hexosamine in $\mathrm{S} \mathrm{XII} \mathrm{is} \mathrm{approximately} 2: 1: 2$.

Methylation studies on $S X I I$. - Gas-liquid chromatography of the alditol acetate derivatives of the $O$-methylglycitols from methylated S XII yielded seven components. The major three components were first to emerge from the column, and corresponded in retention times to authentic 1,5 -di- $O$-acetyl-2,3,4,6-tetra- $O$-methyl- 
TABLE I

RELEASE OF HEXOSAMINE DURING ACID HYDROLYSIS OF S XII ${ }^{\circ}$

Time (h) Hexosamine released (nmoles)

$\begin{array}{ll}1 & 30 \\ 2 & 40 \\ 3 & 41 \\ 4 & 38 \\ 5 & 40 \\ 6 & 35\end{array}$

${ }^{\circ} \mathrm{Hydrolysis}$ of $30 \mu \mathrm{g}$ of S XII with $\mathrm{M}$ hydrochloric acid at $100^{\circ}$.

D-glucitol (3.48 $\mathrm{min}), 1,5$-di- $O$-acetyl-2,3,4,6-tetra- $O$-methyl-D-galactitol (4.36 $\mathrm{min}$ ), and 1,2,5-tri- $O$-acetyl-3,4,6-tri- $O$-methyl-D-glucitol $(6.84 \mathrm{~min})$, respectively. The identities of these three peaks were confirmed by comparison of their mass-spectral analyses with those obtained from authentic standards ${ }^{16}$. Measurement of the areas of the first three peaks indicated a ratio of 0.83:1.00:0.86, respectively. Since only the first three components emerging from the column were shown to be neutral sugars by mass-spectral analyses, this ratio suggests that approximately one half of the D-glucose residues and all of the D-galactose residues are terminal, whereas the remainder of the D-glucose residues are substituted at C-2. The last four components emerging from the column were not further identified.

Similar results were obtained by g.l.c. anaiysis after methanolysis of methylated S XII. Six peaks were obtained. The first and second components emerging from the column (I and $\mathrm{II}$ ) had retention times corresponding to the methyl glycoside(s) of 2,3,4,6-tetra- $O$-methyl-D-glucopyranose $(6.2 \mathrm{~min}$ and $9.0 \mathrm{~min}$ ), the third component (III) to the glycoside(s) of 2,3,4,6-tetra- $O$-methyl-D-galactopyranose (11.2 $\mathrm{min}$ ); and the fourth and fifth components (IV and $V$ ) to the methyl glycoside(s) of 3,4,6-tri- $O$ methyl-D-glucopyranose (13.2 $\mathrm{min}$ and $19.2 \mathrm{~min}$ ). The sixth component $(23.2 \mathrm{~min}$ ) was not identified. The ratio of the sum of the areas of components I, II, IV, and V (methyl glucosides) to the area of component III (methyl galactosides) was 1.70:1.00.

Periodate oxidation of $S X I I$. - Periodate consumption of S XII is shown in Table II. Rapid periodate consumption occurred during the first $8 \mathrm{~h}$ and ceased after 1.65 moles of periodate were consumed per mole of neutral sugar. Carbohydrate analysis indicated the complete destruction of neutral sugar, as previously reported ${ }^{2}$. Loss of neutral sugar would be expected in view of the methylation data, which indicate that each of the neutral sugars has at least one pair of unsubstituted, vicinal hydroxyl groups. A ratio of 1.67 moles of periodate consumed per mole of neutral sugar would be expected assuming a molar ratio of D-glucose to D-galactose of 2:1, with 1 mole of D-glucose and 1 mole of D-galactose residues in nonreducing, terminal positions and the remaining $\mathrm{D}$-glucose residues $(1 \mathrm{~mole})$ substituted at $\mathrm{C}-2$.

Periodate-oxidized, borohydride-reduced S XII did not precipitate rabbit antiXII antiserum, which suggests that periodate oxidation resulted in extensive alteration 
TABLE II

PERIODATE OXIDATION OF S XII ${ }^{a}$

Time $(h) \quad$ Periodate consumed/neutral sugar

(moles/mole)

\begin{tabular}{rl}
\hline 0.1 & 0.45 \\
0.5 & 0.52 \\
1.5 & 0.74 \\
2.5 & 1.15 \\
3.5 & 1.52 \\
4.5 & 1.30 \\
6.5 & 1.52 \\
22 & 1.52 \\
49 & 1.64 \\
71 & 1.60 \\
96 & 1.67
\end{tabular}

${ }^{a}$ With $24 \mathrm{~mm}$ sodium periodate, in the dark at $27^{\circ}$.

of the immunochemical reactivity. Undegraded polysaccharide ( $22 \mu \mathrm{g})$ and oxidizedreduced S XII $(100 \mu \mathrm{g})$ precipitated 32.7 and $1.1 \mu \mathrm{g}$ of antibody nitrogen, respectively. Pre-immunization control antiserum yielded 0.6 and $0.2 \mu \mathrm{g}$ of antibody nitrogen with S XII and oxidized-reduced S XII, respectively.

Oxidation of O-methylalditols of methylated $S$ XII. - Periodate oxidation of L-fucosamine has been shown to produce acetaldehyde, provided that the hydroxyl group at C-4 is unsubstituted ${ }^{14}$. Hydrolysis of methylated S XII yielded a mixture of partially methylated hexoses and hexosamines that, after reduction and periodate oxidation, produced acetaldehyde in an amount equivalent to 0.098 mole of L-fucosamine per mole of neutral sugar. This result indicates that in native S XII a portion of the L-fucosamine residues are substituted at $\mathrm{C}-4$, and consequently, produces, after methylation and hydrolysis, L-fucosamine residues that are unsub-

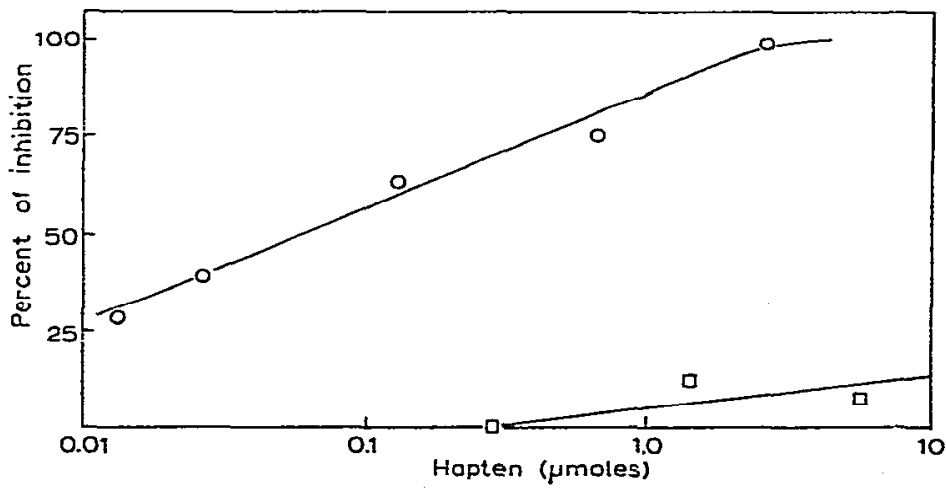

Fig. 1. Inhibition of rabbit anti-XII-dextran-B-1299-S precipitation by kojibiose $(O)$ and by $2-O-\alpha-$ D-galactopyranosyl-D-glucose ( $\square)$. 
stituted at C-4. Previous workers have reported ${ }^{1}$ that the molar ratio of $\mathrm{L}$-fucosamine to neutral sugar in native S XII is $0.33: 1.00$. The low yield of acetaldehyde, representing $30 \%$ of the total L-fucosamine, may be due to destruction of the hexosamine under the conditions of hydrolysis.

Ilapten inhibition of anti-XII and dextran precipitation. - Dextran B-1299-S has been shown to precipitate, from anti-XII antiserum, $50 \%$ of the total antibody nitrogen that may be precipitated by the homologous polysaccharide ${ }^{3}$. The inhibition by kojibiose and 2-O- $\alpha$-D-galactopyranosyl-D-glucose of the precipitation of rabbit anti-XII antiserum and dextran B-1299-S is shown in Fig. 1. Addition of $0.06 \mu$ mole of kojibiose produced a $50 \%$ inhibition, whereas $5.6 \mu$ moles of $2-O-\alpha$-D-galactopyranosyl-D-glucose inhibited precipitation by only $10 \%$. The disaccharide structure, 2-O- $\alpha$-D-galactopyranosyl-D-glucose, may be present in S XII, but it does not contribute to the cross reaction between anti-XII and dextran B-1299-S.

DISCUSSION

A molar ratio of D-glucose to D-galactose of approximately 2:1 was obtained by direct analysis of the products of acid hydrolysis of S XII and by determination of the methyl ethers of the neutral sugars obtained by acetolysis of methylated S XII. The molar ratio of hexose to hexosamine was found to be $\sim 3: 2$.

The absence of dimethyl ethers of neutral sugars in the acetolyzate of methylated S XII suggests that methylation of the polysaccharide was complete and that neutral sugars did not serve as branch points in the polysaccharide structure. Further, these ratios suggest that the backbone of the polysaccharide probably contains hexosamine residues that are each substituted at a position other than that involved in the backbone linkage. The formation of acetaldehyde by periodate oxidation of hydrolyzates of methylated S XII indicates that at least a portion of the L-fucosamine residues of S XII are substituted at C-4. Such substitution is consistent with a hexosamine backbone in which L-fucosamine residues are substituted at C-3 and C-4.

Fig. 2 shows a tentative model for the structural repeating unit of S XII. This structure conforms to the results of methylation data and periodate oxidation. An

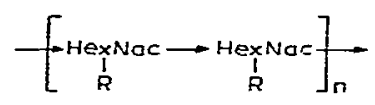

HexNac $=2$-acetcmido-2-deoxy-D-galactose

or 2-acetamido-2,6-dideoxy-L-galactose

$R=\alpha-0-G i=p-(1 \rightarrow 2)-G i c \rho$ or $D-G a l s$

Fig. 2. Suggested repeating units of S XII.

important feature of the model is the linear chain of glycosidically-linked hexosamines. The present investigation has shown that oxidized-reduced S XII does not cross-react with type XII antiserum, although the hexosamine residues appear to be intact. The location of the hexosamine residues in the interior of the molecule, and the high degree of substitution may account for their weak immunochemical properties. 
The identification of 2,3,4,6-tetra- $O$-methyl-D-galactopyranose as the only $D$-galactose derivative from the hydrolyzate of methylated S XII indicates that all the $D$-galactose residues occur at nonreducing termini. However, $\alpha$-galactosidases from Phaseolus vulgaris ${ }^{17}$ and Aspergillus niger ${ }^{18}$, a broad-specificity $\beta$-galactosidase from bovine testes ${ }^{19}$, and an $\alpha$-D-galactosyl-binding lectin from Bandeirea simplicifolia ${ }^{20}$ have failed to confirm the presence of the nonreducing terminal D-galactosyl groups. Steric effects of adjacent groups on S XII may be interfering with the binding of these $D$-galactosyl-specific proteins to the nonreducing $D$-galactosyl groups or labile blocking groups at the C-6 position of D-galactose may be present. Such blocking groups would remain undetected by methylation analysis of S XII and would not interfere with periodate oxidation. The presence, in $S \mathrm{XII}$, of the disaccharide structure $2-O-\alpha$-D-galactopyranosyl-D-glucose is consistent with all the structural chemical data reported but, if present, it does not appear to be immunochemically important in the cross-reaction between dextran and anti-XII antiserum. On the basis of all available information, we conclude that the side-chains consist of D-galactopyranosyl and $O$ - $\alpha$-D-glucopyranosyl-(1 $\rightarrow 2)$-D-glucopyranosyl residues.

A more complete understanding of the structure of S XII awaits the isolation of any $O$-methyl- $N$-methylhexosamines from the hydrolyzate of methylated S XII. Work on the structure of the hexosamine backbone and the immunochemical significance of the terminal D-galactose residues is in progress.

\section{ACKNOWLEDGMENTS}

We thank Drs. G. Jourdian and J. Distler for assistance in the quantitative determination of periodate consumption and in the preparation of this manuscript. In addition, the valuable assistance of Dr. A. Misaki in the methylation of the polysaccharide and g.l.c. is acknowledged. This work was supported in part by a research grant (AI-AM-10171) from the National Institutes of Allergy and Immunology and of Arthritis, Metabolism, and Digestive Diseases, U.S. Public Health Service and by a U.S.P.H.S. training grant (GM-00187).

\section{REFERENCES}

I J. A. Cifonelli, P. Rebers, M. B. PerRy, AND J. K. N. Jones, Biochemistry, 5 (1966) 3066-3072.

2 I. J. Goldstein, J. A. Cifonelli, ANd J. Duke, Biochemistry, 13 (1974) 867-870.

3 J. W. Goodman ANd E. A. Kabat, J. Immunol., 84 (1960) 347-357.

4 H. SuzuKI ANd E. J. HeHre, Arch. Biochem. Biophys., 104 (1964) 305-313.

5 M. Dubois, K. A. Gilles, J. K. Hamilton, P. A. Rebers, and F. Smith, Anal. Chem., 28 (1956) 350-356.

6 C. J. M. Rondle AND W. T. J. MoRGAN, Biochem. J., 61 (1955) 586-589.

7 J. A. Cifonelli And J. King, Carbohyd. Res., 12 (1970) 391-402.

8 D. H. Campbell, J. S. Garvey, N. E. Cremer, and D. H. Sussdorf, Methods in Immunology, W. A. Benjamin, New York, 1963, sections B-4a and B-5a.

9 P. A. SANDFORd AND H. E. CoNRAd, Biochemistry, 5 (1966) 1508-1517.

10 K. Stellner, H. Sarto, and S. Hakomori, Arch. Biochem. Biophys., 155 (1973) 464-472.

11 A. Misakt, T. Ito, and T. Harada, Agr. Biol. Chem. (Tokyo), 36 (1972) 761-771.

12 G. O. Aspinall, J. Chem. Soc., (1963) 1676-1684. 
13 E. A. KABAT, Kabat and Mayer's Experimental Immunochemistry, Thomas, Springfield, 1961, pp. 547-548.

14 A. K. Bhattacharyya AND D. AMinoff, Anal. Biochem., 14 (1966) 278-289.

15 H. RosEN, Arch. Biochem. Biophys., 67 (1957) 10-15.

16 B. Lindere, Methods Enzymol, 28 (1972) 178-195.

17 O. P. Bahi and K. M. L. Agrawali, J. Biol. Chem., 243 (1968) 98-102.

18 O. P. Bahl and K. M. L. Agrawall, J. Biol. Chem., 244 (1969) 2970-2978.

19 J. J. Distuer And G. W. Jourdian, J. Biol. Chem., 248 (1973) 6772-6780.

20 C. E. Hayes and I. J. Goldstern, J. Biol. Chem., 249 (1974) 1904-1914. 